\title{
Gluing Kuramoto coupled oscillators networks
}

\author{
Eduardo Canale and Pablo Monzón
}

\begin{abstract}
In this work we prove that the problem of almost global synchronization of the Kuramoto model of sinusoidally symmetric coupled oscillators with a given topology could be reduced to the analysis of the blocks of the underlying interconnection graph.
\end{abstract}

\section{INTRODUCTION}

A few decades ago, Y. Kuramoto introduced a mathematical model of weakly coupled oscillators that gave a formal framework to some of the works of A.T. Winfree on biological clocks [1], [2], [3]. The model proposes the idea that several oscillators can interact in a way such that the individual oscillation properties change in order to achieve a global behavior for the interconnected system. The Kuramoto model serves a a good representation of many systems in several contexts such a biology, engineering, physics, mechanics, etc. [4], [5], [6], [7], [8], [9], [10], [11], [12], [13], [14].

Recently, many works on the control community have focused on the analysis of the Kuramoto model, specially the one with sinusoidal coupling. The consensus or collective synchronization of the individuals is particularly important in many applications that need to represent coordination, cooperation, emerging behavior, etc. Local stability properties of the consensus have been initially explored in [15], while global or almost global dynamical properties were studied in [16], [17], [18]. In these works, the relevance of the underlying graph describing the interconnection of the system was hinted. In the present article, we go deeper on the analysis of the relationships between the dynamical properties of the system and the algebraic properties of the interconnection graph, exploiting the strong algebraic structure that every graph has.

In Section II we quickly review the relevant aspects of the algebraic graph theory. After that, we summarize the main results of different previous works on the analysis of Kuramoto coupled oscillators. Section IV contains the contributions of this article, showing how we can interconnect synchronized systems keeping the synchronization property and introducing an analysis procedure for a kind of graphs. Finally, we present some conclusions.

This work was partially supported by CSIC-UDELAR

Pablo Monzón is with the Electrical Engineering Institute, Faculty of Engineering, Universidad de la Republica, Uruguay, monzonefing.edu.uy

Eduardo Canale is with the Mathematics and Statistics Institute (IMERL), Faculty of Engineering, Universidad de la Republica, Uruguay, canaledfing.edu.uy

\section{Algebraic GRAPH THEORY}

In this Section we review the basic facts on algebraic graph theory that will be used along the article. A more detailed introduction to this theory can be found in [19], [20]. A graph consists in a set of $n$ nodes or vertices $V G=\left\{v_{1}, \ldots, v_{n}\right\}$ and a set of $m$ links or edges $E G=\left\{e_{1}, \ldots, e_{m}\right\}$ that describes how the nodes are related to each other. If $n=$ 1 the graph is called trivial. We say that two nodes are neighbors or adjacent if there is a link in $E G$ between them. If all the vertices are pairwise adjacent the graph is called complete and written $K_{n}$. A walk is a sequence $v_{0}, \ldots, v_{n}$ of adjacent vertices. If the vertices are different except the first and the last which are equal $\left(v_{i} \neq v_{j}\right.$ for $0<i<j$ and $v_{0}=v_{n}$ ) the walk is called a cycle. A graph with no cycle is called acyclic. The graph is connected if there is a walk between any given pair of vertices. A tree is an acyclic connected graph and has $m=n-1$ edges. The graph is oriented if every link has a starting node and a final node. The topology of a oriented graph may be described by the incidence matrix $B$ with $n$ rows and $m$ columns:

$$
B_{i j}=\left\{\begin{array}{cl}
1 & \text { if edge } j \text { reaches node } i \\
-1 & \text { if edge } j \text { leaves node } i \\
0 & \text { otherwise }
\end{array}\right.
$$

Observe that ${ }^{1} B^{T} \mathbf{1}_{n}=0$. The vertex space and the edge space of $G$ are the sets of real functions with domain $V G$ and $E G$ respectively, which we sometimes will identify, respectively, with the vectors sets $\mathbb{R}^{n}$ and $\mathbb{R}^{m}$. Thus, the incidence matrix $B$ can be seen as a linear transformation from the edge space to the vertex space. The kernel of $B$ is the cycle space of the graph $G$ and its elements are called flows. Every flow can be thought as a vector of weights assigned to every link in a way that the total algebraic sum at each node is zero. The cycle space is spanned by the flows determined by the cycles: given a cycle $v_{0}, \ldots, v_{n}=v_{0}$, its associated flow $f_{C}(e)$ is \pm 1 if $e$ leaves some $v_{i}$ and reaches $v_{i \pm 1}$ and 0 otherwise.

If the graph $G$ is the union of two nontrivial graphs $G_{1}$ and $G_{2}$ with one and only one node $v_{i}$ in common, then $v_{i}$ is called a cut-vertex of $G$. A connected graph with more than two vertices and no cut-vertex is called 2-connected and it follows that for every pair of nodes, there are at least two different paths between them. Given a subset $V_{1} \subset V G$, its induced subgraph is $\left\langle V_{1}\right\rangle$ with vertex set $V_{1}$ and edge set $\left\{e \in E G: e\right.$ joins vertices of $\left.V_{1}\right\}$. The maximal induced \footnotetext{
to 1 .

${ }^{1}$ By $\mathbf{1}_{p}$ we denote the column vector in $\mathbb{R}^{p}$ with all its components equal
} 
subgraphs of $G$ with no cut-vertex, are called the blocks of $G$. Every graph has the form of figure 1: a collection of blocks joined by cut-vertices. For a complete graph, there is only one block, the graph itself.

We will use the following vector notation: given

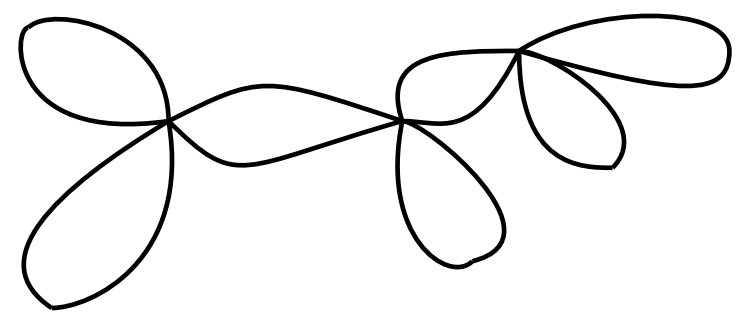

Fig. 1. Representation of a graph as a union of blocks.

a $n$-dimensional vector $\bar{\theta}=\left[\theta_{1}, \ldots, \theta_{n}\right]$, then $\bar{\theta}(i: j)=\left[\theta_{i}, \ldots, \theta_{j}\right]$ and $\bar{\theta}(i)=\theta_{i}$. Firstly, we present some basic results. We include two different proofs for Lemma 2.1, in order to show two distinct interpretations of the same facts: one based on linear algebra, the other using graph theory elements.

Lemma 2.1: Consider a graph $G$, with $v$ a cut-vertex between $G_{1}$ and $G_{2}$. Then, an edge space element $f: E G \rightarrow$ $\mathbb{R}$ is a flow on $G$, if and only if $\left.f\right|_{E G_{1}}$ and $\left.f\right|_{E G_{2}}$ are a flows on $G_{1}$ and $G_{2}$ respectively.

Proof 1: Suppose that the $i$ vertices of $G_{1}$ and its $k$ edges come first in the chosen labelling. Suppose, also, that $v=v_{i}$, then $B$ has the following form:

$$
B=\left[\begin{array}{c|c}
W_{1} & \mathbf{0}_{(\mathbf{i}-\mathbf{1}) \times(\mathbf{m}-\mathbf{k})} \\
\hline w_{1}^{T} & w_{2}^{T} \\
\hline \mathbf{0}_{(\mathbf{n}-\mathbf{i}) \times \mathbf{k}} & W_{2}
\end{array}\right]
$$

Where $w_{1}$ and $w_{2}$ are column vectors with appropriate dimensions. With this notation, the incidence matrices of $G_{1}$ and $G_{2}$ are, respectively

$$
B_{1}=\left[\frac{W_{1}}{w_{1}^{T}}\right], \quad B_{2}=\left[\frac{w_{2}^{T}}{W_{2}}\right] .
$$

Besides, $B_{1}$ as incidence matrix, verifies $\mathbf{1}_{i}^{T} B_{1}=0$, thus $\mathbf{1}_{i-1}^{T} W_{1}+w_{1}^{T}=\mathbf{0}_{k}$, so

$$
w_{1}^{T}=-\mathbf{1}_{i-1}^{T} W_{1} .
$$

Let $f$ be a flow on $G$. Then, in order to prove that $f_{1}=$ $\left.f\right|_{E G_{1}}$ is a flow on $G_{1}$, we must prove that $B_{1} f_{1}=\mathbf{0}_{i}$, i.e. $W_{1} f_{1}=\mathbf{0}_{i-1}$ and $w_{1}^{T} f_{1}=0$. The former is true because $f$ is a flow on $G$, thus $B f=\mathbf{0}_{n}$, but $W_{1} f_{1}=$ $(B f)(1: i-1)$. While for the last, we have that, by (1), $w_{1}^{T} f_{1}=\left(-\mathbf{1}_{i-1}^{T} W_{1}\right) f_{1}=-\mathbf{1}_{i-1}^{T}\left(W_{1} f_{1}\right)=\mathbf{1}_{i-1}^{T} \mathbf{0}_{i-1}=0$. Exchanging $G_{1}$ by $G_{2}$ we obtain that $\left.f\right|_{E G_{2}}$ is a flow on $G_{2}$.

Conversely, if $f_{1}$ and $f_{2}$ are flows on $G_{1}$ and $G_{2}$ respectively, we have that $(B f)(1: i-1)=B_{1} f_{1}=\mathbf{0}_{i},(B f)(i+1: n)=$ $B_{2} f_{2}=\mathbf{0}_{n-i+1}$ and $(B f)(i)=w_{1}^{T} f_{1}+w_{2}^{T} f_{2}=0+0=0$.

Proof 2: Following [19] [Lemma 5.1, Theorem 5.2], given a spanning tree $T$ of $G$, we obtain a basis of the cycle space in the following form: for each edge $e \in E^{\prime}=E G \backslash E T$ we have an unique cycle $\operatorname{cyc}(T, e)$ which determines a flow $f_{T, e}$. The set $\mathcal{B}$ of these flows is a basis of the cycle-space. However, since $v$ is a cut-vertex, any cycle is included either in $G_{1}$ or in $G_{2}$, so its associated flow is null either in $E G_{1}$ or in $E G_{2}$. If we regard a flow on $G$ which is null in $E G_{1}$ as a flow on $G_{2}$, we can split $\mathcal{B}$ into two sets $\mathcal{B}_{1}$ and $\mathcal{B}_{2}$ cycle-space basis of $G_{1}$ and $G_{2}$ respectively. Thus the cyclespace of $G$ is the direct sum of the cycle-spaces of $G_{1}$ and $G_{2}$.

Lemma 2.2: Let $G$ be a graph, $V_{1} \subset V G$ and $G_{1}=\left\langle V_{1}\right\rangle$ the subgraph of $G$ induced by the vertices $V_{1}$ with incidence matrix $B_{1}$. Let $H: \mathbb{R} \rightarrow \mathbb{R}$ be any real function, $\bar{\theta}: V \rightarrow \mathbb{R}$ an element of the vertex-space of $G$ and $f=H\left(B^{T} \bar{\theta}\right)$ then, if

$$
f_{1}=\left.f\right|_{E G_{1}}, \quad \bar{\theta}_{1}=\left.\bar{\theta}\right|_{V G_{1}}
$$

it is true that

$$
f_{1}=H\left(B_{1}^{T} \bar{\theta}_{1}\right)
$$

Proof: Suppose that the $i$ vertices and $k$ edges of $G_{1}$ come first in the chosen labelling. Then, for some $B^{\prime}, B^{\prime \prime}$ and $\bar{\theta}_{2}$, we have that

$$
B^{T} \bar{\theta}=\left[\begin{array}{c|c}
B_{1}^{T} & \mathbf{0}_{\mathbf{i} \times \mathbf{k}} \\
\hline B^{\prime} & B^{\prime \prime}
\end{array}\right]\left[\begin{array}{c}
\bar{\theta}_{1} \\
\bar{\theta}_{2}
\end{array}\right]=\left[\begin{array}{c}
B_{1}^{T} \bar{\theta}_{1} \\
B^{\prime} \bar{\theta}_{1}+B^{\prime \prime} \bar{\theta}_{2}
\end{array}\right] .
$$

Thus, $\left(B^{T} \bar{\theta}\right)(1: k)=B_{1}^{T} \bar{\theta}_{1}$, and $f_{1}=f(1: k)=$ $H\left(B^{T} \bar{\theta}\right)(1: k)=H\left(\left(B^{T} \bar{\theta}\right)(1: k)\right)=H\left(B_{1}^{T} \bar{\theta}_{1}\right)$.

\section{AlmOST GLOBAL SYNCHRONIZATION}

Oscillators have been studied by engineers for a long time [21]. The state of an oscillator can be described by its phase angle $\theta$. Consider now the Kuramoto model of $n$ sinusoidally coupled oscillators [6]

$$
\dot{\theta}_{i}=\sum_{j \in \mathcal{N}_{i}} \sin \left(\theta_{j}-\theta_{i}\right) \quad i=1, \ldots, n
$$

where $\mathcal{N}_{i}$ is the set of neighbors of agent $i$. Each phase $\theta_{i}$ belongs to the interval $[0,2 \pi)$, so the system evolves on the compact $n$-dimensional torus $\mathcal{T}^{n}$. The value of a phase must be considered modulo $2 \pi$. Consider the graph $G$, with nodes $\left\{v_{1}, \ldots, v_{n}\right\}$ and edges $\left\{e_{1}, \ldots, e_{m}\right\}$, that describes how the individual oscillators, or agents, interact between each other. The node $v_{i}$ represents the $i$-th oscillator, with phase $\theta_{i}$. Consider an arbitrary orientation of the links of $G$ and let the matrix $B$, with $n$ rows and $m$ columns, be an incidence matrix for $G$. We will work with symmetric interaction: if 
$i \in \mathcal{N}_{j}$ then $j \in \mathcal{N}_{i}$. In this case, as is explained in [15], the expression (2) can be compactly written as

$$
\dot{\theta}=-B \sin \left(B^{T} \bar{\theta}\right)
$$

Equation (3) does not depend on the choice of $B$. As was done by Kuramoto, we may represent the agents as running points on a circumference or as unit phasors, as in Example 3.1 [1], [17].

Since the system dynamic depends only on the phase differences, it is invariant under translations parallel to vector $\mathbf{1}_{n}$. We say the system synchronizes or reaches consensus if the individual phases converge to a state where all the phases are identical. Of course, a consensus point is an equilibrium point of the system and actually we have a synchronization set, due to the invariance property. This also applies to every equilibrium point. We will also work with partial consensus equilibria, when most of the phases take the value 0 (taking a suitable reference) and the remaining phases take the value $\pi$. Other equilibria will be referred as non-synchronized. If $\bar{\theta}$ is an equilibrium point of (3) with underlying graph $G$, we will use the expression: $\bar{\theta}$ is an equilibrium of $G$.

Example 3.1: Consider the graph shown at the left of Figure 2. A non-synchronized equilibrium point is given by

$$
\bar{\theta}=\left[\begin{array}{c}
169.04 \\
59.96 \\
-49.13 \\
130.87 \\
-120.04 \\
-10.96 \\
-30.04 \\
149.96
\end{array}\right]
$$

The angles are measured in degrees.

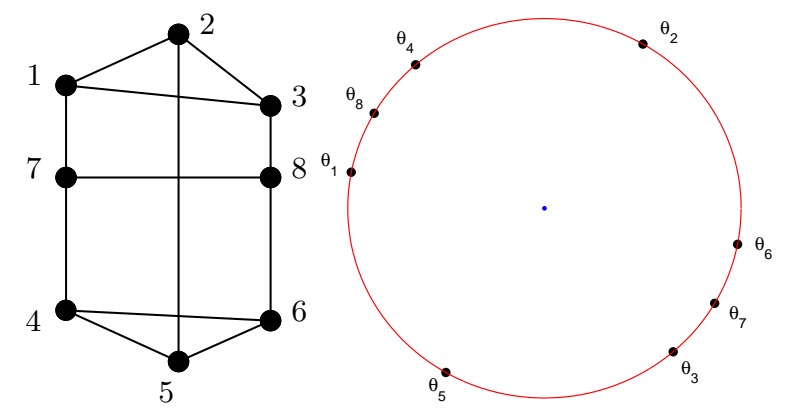

Fig. 2. Phasor representation of the equilibrium point $\bar{\theta}$ of system (3) of Example 3.1. The underlying graph is shown at the left.

We are concerned on whether or not all the trajectories converge to the synchronization set. Since the system has many equilibria, we can only expect that most of the trajectories presents this property. Following the ideas of [22], we say that the system has the almost global synchronization property if the set of trajectories that do not converge to the synchronization set has zero Lebesgue measure on $\mathcal{T}^{n}$. If the system is described by a graph $G$, we will shortly say the $G$ is a.g.s.. In [15], it was proved that the synchronization set is locally stable. First results on almost global properties were presented in [16], [18]. There, it was proved that the complete graph $K_{n}$ and the tree graphs always are a.g.s. These results were proved in two steps: firstly, using LaSalle's result on asymptotical behavior of trajectories in a compact invariant set, it was shown that the only $\omega$-limit sets are the equilibria of the system; secondly, Jacobian linearization was used to locally classify the equilibria ${ }^{2}$ [23]. At an equilibrium point $\bar{\theta}$, a first order approximation is given by the symmetric, $n \times n$, matrix

$$
A_{G}=-B \operatorname{diag}\left[\cos \left(B^{T} \bar{\theta}\right)\right] B^{T}
$$

Observe that $A_{G}$ always has a zero eigenvalue with corresponding eigenvector 1 , due to the invariance property of the system. In this work, we try to extend our knowledge of the family of a.g.s. graphs.

\section{SYNCHRONIZING INTERCONNECTION}

From equation (3) we see that a phase angle $\theta$ is an equilibrium point if and only if $\sin \left(B^{T} \theta\right)$ is a flow on $G$. Thus it should be possible that this equilibrium points could be obtained from the equilibrium points of the blocks of the graphs. In fact, this is exactly what happens. Furthermore, the stability of these equilibrium points depends only on the stability of the associated equilibrium points of the blocks. First, we study the existence problem, which will follow directly from Lemma 2.1, and then we study the stability properties.

\section{A. Existence}

If $\theta_{1}: V G_{1} \rightarrow \mathbb{R}$ is in the vector space of a subgraph $G_{1}$ of $G$, we will regard it also as its unique extension to the vector space of $G$ which is null elsewhere of $G_{1}$. The same for an element of the edge space.

Proposition 4.1: Consider the graph $G$ with a cut-vertex $v$ between $G_{1}$ and $G_{2}$. If $\bar{\theta}$ is an equilibrium point of $G$, then $\bar{\theta}_{1}=\left.\bar{\theta}\right|_{V G_{1}}$ and $\bar{\theta}_{2}=\left.\bar{\theta}\right|_{V G_{2}}$ are equilibrium points of $G_{1}$ and $G_{2}$ respectively. Conversely, if $\bar{\theta}_{1}$ and $\bar{\theta}_{2}$ are equilibrium points of $G_{1}$ and $G_{2}$ respectively, there exists a real number $\alpha$, such that $\bar{\theta}_{2}^{\prime}=\bar{\theta}_{2}+\alpha$ is an equilibrium point of $G_{2}$ and $\bar{\theta}=\bar{\theta}_{1}+\bar{\theta}_{2}^{\prime}$ is an equilibrium point of $G$.

Proof: Let $B, B_{1}, B_{2}$, etc. like in Lemma 2.1. If $\bar{\theta}$ is an equilibrium point of $G$, then $f=\sin \left(B^{T} \bar{\theta}\right)$ is a flow on $G$, thus, by Lemma 2.1, $f_{1}=\left.f\right|_{V G_{1}}$ is a flow on $G_{1}$. Thus, it is enough to prove that $f_{1}=\sin \left(B_{1}^{T} \bar{\theta}_{1}\right)$, which follows from Lemma 2.2, taking $H(x)=\sin (x)$ and noticing that $G_{1}$ is an induced subgraph of $G$. The case for $G_{2}$ follows by the symmetry between $G_{1}$ and $G_{2}$.

\footnotetext{
${ }^{2}$ Almost global synchronization could be proved via density functions only for the cases of 2 and 3 agents [17].
} 
Now, assume that $\bar{\theta}_{1}$ and $\bar{\theta}_{2}$ are equilibrium points of $G_{1}$ and $G_{2}$ respectively. Let $\alpha=\bar{\theta}_{1}(v)-\bar{\theta}_{2}(v), \bar{\theta}_{2}^{\prime}=\bar{\theta}_{2}+\alpha$, $\bar{\theta}=\bar{\theta}_{1}+\bar{\theta}_{2}^{\prime}$ and $f=\sin \left(B^{T} \bar{\theta}\right)$. Then, by Lemma 2.2 , $f_{1}=\left.f\right|_{E G_{1}}=\sin \left(B_{1}^{T} \bar{\theta}_{1}\right)$ and $f_{2}=\left.f\right|_{E G_{2}}=\sin \left(B_{2}^{T} \bar{\theta}_{2}^{\prime}\right)$. On the other hand, due to the invariance of the system we have remarked on Section III, the vector $\bar{\theta}_{2}^{\prime}$ is also an equilibrium point of $G_{2}$, and then, $f_{1}$ and $f_{2}$ are flows in $G_{1}$ and $G_{2}$ respectively. Therefore, by Lemma $2.1, f_{1}+f_{2}$ is a flow on $G$, but $f=f_{1}+f_{2}$, because $E G_{1} \cap E G_{2}=\emptyset$.

\section{B. Stability analysis}

We will relate the stability properties of the graph $G$ with a cut-vertex with the stability properties of the subgraphs $G_{1}$ and $G_{2}$ joined by it. Since every equilibrium of $G$ defines an equilibria for $G_{1}$ and $G_{2}$, we wonder whether or not the dynamical characteristics of these equilibria are or not the same. We will use Jacobian linearization. The zero eigenvalue is always present due to the invariance of the system by translations parallel to $\mathbf{1}_{\mathbf{n}}$. We always refer to the transversal stability of the equilibrium set. If the multiplicity of the zero eigenvalue is more than one, Jacobian linearization may fail in classifying the equilibria. Due to space reasons, we present the study of this particular problem in a different article. So, in this work, we assume that we always have a single null eigenvalue.

Theorem 4.1: Consider the graph $G$, with a cut-vertex $v$ joining the subgraphs $G_{1}$ and $G_{2}$ of graph $G$. Let $\bar{\theta} \in \mathbb{R}^{n}$ be an equilibrium point of $G$. Then, $\bar{\theta}$ is locally stable if and only if $\bar{\theta}_{1}=\left.\bar{\theta}\right|_{V G_{1}}$ and $\bar{\theta}_{2}=\left.\bar{\theta}\right|_{V G_{2}}$ are locally stable and coincide in $v\left(=V G_{1} \cap V G_{2}\right)$.

Proof: Recall that the first order approximation of the system around an equilibrium point is given by

$$
A_{G}=-B \operatorname{diag}\left[\cos \left(B^{T} \bar{\theta}\right)\right] B^{T} .
$$

Suppose that $G_{1}$ has $i$ vertices, that they come first in the chosen labelling and that $v$ is the last of them $\left(v=v_{i}\right)$. Then, a direct calculation gives

$$
A_{G}=A_{1}+A_{2},
$$

with

$$
A_{1}=\left[\begin{array}{l|l}
A_{G_{1}} & \mathbf{0}_{\mathbf{i} \times(\mathbf{n}-\mathbf{i})} \\
\hline \mathbf{0}_{(\mathbf{n}-\mathbf{i}) \times \mathbf{i}} & \mathbf{0}_{(\mathbf{n}-\mathbf{i}) \times(\mathbf{n}-\mathbf{i})}
\end{array}\right]
$$

and

$$
A_{2}=\left[\begin{array}{l|l}
\mathbf{0}_{(\mathbf{i}-\mathbf{1}) \times(\mathbf{i}-\mathbf{1})} & \mathbf{0}_{(\mathbf{i}-\mathbf{1}) \times(\mathbf{n}-\mathbf{i}+\mathbf{1})} \\
\hline \mathbf{0}_{(\mathbf{n}-\mathbf{i}+\mathbf{1}) \times(\mathbf{i}-\mathbf{1})} & A_{G_{2}}
\end{array}\right] .
$$

Observe that these matrices partially overlap, so the matrix $A$ takes the form:

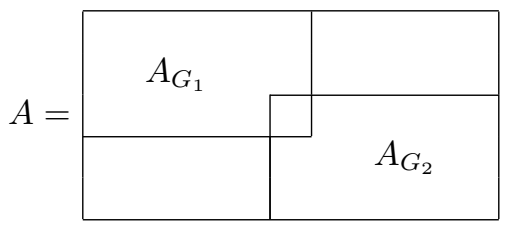

First of all, we consider the case with $\bar{\theta}_{1}$ and $\bar{\theta}_{2}$ stable and $\bar{\theta}_{1}(i)=\bar{\theta}_{2}(i)$. Then, $A_{G_{1}}$ and $A_{G_{2}}$ are stable and equation (5) holds for $\bar{\theta}=\left[\bar{\theta}_{1}, \bar{\theta}_{2}(2: n-i)\right]$. So, $A_{G}$ is the sum of two semidefinite negative matrices which gives rise a semidefinite negative one. Besides, the kernel of $A_{G}$ has dimension 1 , since if $A_{G} w=0$ then $w^{T} A_{G} w=0$, thus, $w^{T} A_{1} w+w^{T} A_{2} w=0$. But, $w^{T} A_{1} w=w_{1}^{T} A_{G_{1}} w_{1}$ and $w^{T} A_{2} w=w_{2}^{T} A_{G_{2}} w_{2}$ for $w_{1}=\left.w\right|_{V G_{1}}$ and $w_{2}=\left.w\right|_{V G_{2}}$. Then $w_{1}^{T} A_{G_{1}} w_{1}+w_{2}^{T} A_{G_{2}} w_{2}=0$. That can happen if only if $w_{1}^{T} A_{G_{1}} w_{1}=0$ and $w_{2}^{T} A_{G_{2}} w_{2}=0$. But the kernels of $A_{G_{1}}$ and $A_{G_{2}}$ are spanned by $\mathbf{1}_{\mathbf{i}}$ and $\mathbf{1}_{\mathbf{n}-\mathbf{i}+\mathbf{1}}$ respectively, thus $w_{1}=\alpha \mathbf{1}_{\mathbf{i}}$ and $w_{2}=\beta \mathbf{1}_{\mathbf{n}-\mathbf{i}}$. But $w_{1}(i)=w_{2}(1)=w(i)$, thus $\alpha=\beta$ and $w=\alpha \mathbf{1}_{n}$. This proves the stability of $A_{G}$.

Now, we focus on the case with $\bar{\theta}_{1}$ or $\bar{\theta}_{2}$ unstable. We analyze the first case, since the other is similar. Suppose that $A_{G_{1}}$ has a positive eigenvalue with associated eigenvector $w_{1}$, thus

$$
w_{1}^{T} A_{G_{1}} w_{1}>0 .
$$

Define the vector

$$
w=\left[\begin{array}{c}
w_{1} \\
w_{1}(i) \mathbf{1}_{\mathbf{n}-\mathbf{i}}
\end{array}\right]=\left[\begin{array}{c}
w_{1}(1: i-1) \\
w_{1}(i) \mathbf{1}_{\mathbf{n}-\mathbf{i}+\mathbf{1}}
\end{array}\right] .
$$

Then,

$$
w^{T} A_{G} w=w_{1}^{T} A_{G_{1}} w_{1}+w_{1}(i)^{2} \mathbf{1}_{\mathbf{n}-\mathbf{i}+\mathbf{1}}{ }^{T} A_{G_{2}} \mathbf{1}_{\mathbf{n}-\mathbf{i}+\mathbf{1}}
$$

which actually is $w_{1}^{T} A_{G_{1}} w_{1}>0$ since $A_{G_{2}} \mathbf{1}_{\mathbf{n}-\mathbf{i}+\mathbf{1}}=0$. Then, $\bar{\theta}$ is unstable.

We are now ready to state and prove the main result of this article.

Theorem 4.2: Consider the graph $G$, with a cut-vertex $v_{i}$ joining the subgraphs $G_{1}$ and $G_{2}$. Then, $G_{1}$ and $G_{2}$ have the almost global synchronization property if and only if $G$ does.

Proof: First of all, let $\bar{\theta}$ be an equilibrium point of $G$. According to Theorem 4.1, $\bar{\theta}$ is stable only if $\bar{\theta}_{1}=\left.\bar{\theta}\right|_{V G_{1}}$ and $\bar{\theta}_{2}=\left.\bar{\theta}\right|_{V G_{2}}$ are too.

If $G_{1}$ and $G_{2}$ are a.g.s., the only locally stable set is the consensus, and since they have a vertex in common, the only locally stable equilibria of $G$ is also the consensus and $G$ is a.g.s.

In the other direction, if $\bar{\theta}_{1}$ is a locally stable equilibrium of $G_{1}$, we chose $\bar{\theta}=\left[\bar{\theta}_{1}, \bar{\theta}_{1}(i) \mathbf{1}_{\mathbf{n}-\mathbf{i}}\right]$ and we construct a stable equilibrium for $G$ (as we have mentioned before, a consensus equilibrium is always locally stable [15]). Since $G$ is a.g.s., $\bar{\theta}$, and so $\bar{\theta}_{1}$, must be consensus equilibrium points. 
Theorem 4.2 has many direct consequences. We point out some of them, with a brief hint of the respective proofs.

Proposition 4.2: Consider a graph $G$ with a bridge $e_{k}$ between the nodes $v_{i}$ and $v_{j}$ and let $G_{1}$ and $G_{2}$ be the connected components of $G \backslash\left\{e_{k}\right\}$. Then, $G$ is a.g.s. if and only if $G_{1}$ and $G_{2}$ are.

If a graph has a bridge, i.e., an edge whose removal disconnect the graph, the behavior of the system depends only on the parts connected by the bridge. Indeed, the bridge together with its ends vertices form a block, which is in fact a complete graph and its vertices are cut-vertices of the graph, as is shown in figure 3. Since any complete graph is a.g.s., the a.g.s. character of the original graph depends on the other blocks.

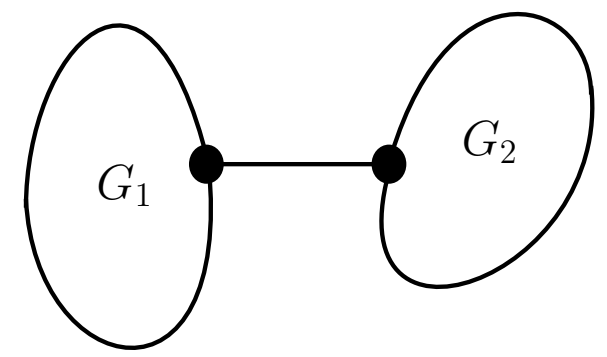

Fig. 3. A graph with a bridge.

Theorem 4.3: A graph $G$ is a.g.s. if and only if every block of $G$ is a.g.s.

The graph $G$ can be partitioned into its blocks. Then, $G$ can be thought as a collection of subgraphs connected by cut-vertices. An iterative use of Theorem 4.2 leads us to the result. Observe that Theorem 4.3 reduces the characterization of the family of a.g.s. graphs to the analysis of 2-connected graphs. As an application, consider the case where we connect two a.g.s. graphs through another a.g.s. graph. In this way, we construct a new a.g.s. graph. Figures 4 and 5 illustrate the situation.

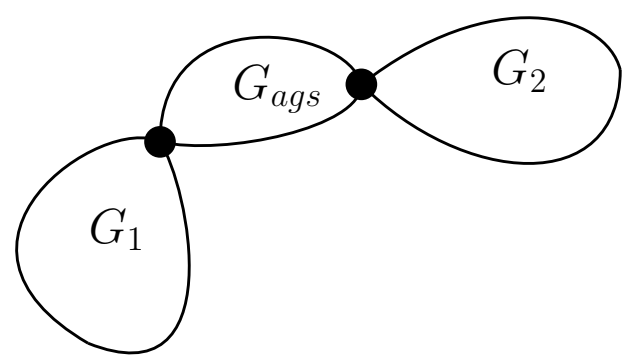

Fig. 4. Two graphs connected by an a.g.s. graph.

In [16], it was proved the next result

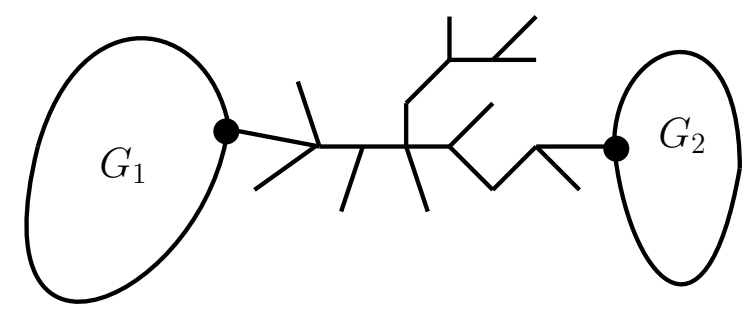

Fig. 5. Two graphs connected by a tree

Proposition 4.3: If $G$ is a tree, it is always a.g.s.

The proof was done using a colouring technique at all the equilibria. Now, we have two alternatives proofs. The first one using Theorem 4.3. We observe that a the blocks of a tree are all $K_{2}$, and then, they are a.g.s. The second one is applying iteratively Proposition 4.2, since every link of a tree is a bridge.

If we have a graph with arboricities, like the one shown in figure 6, we can neglect the trees in order to prove the a.g.s. property.

Corollary 4.1: A graph with the structure shown in figure 6 is a.g.s. if and only if $G_{1}$ is.

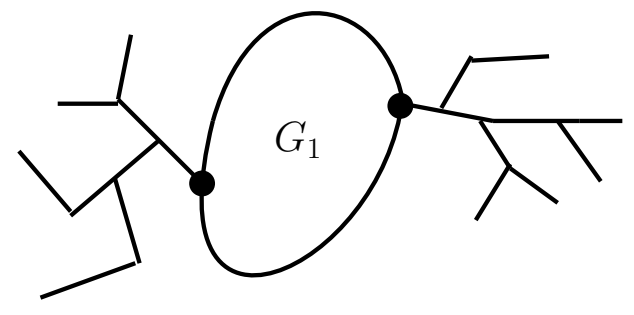

Fig. 6. A graph with arboricities.

To conclude this section, we present two general methods for constructing a.g.s. systems.

Proposition 4.4: If $G$ is a tree and we build a new graph $K$ replacing some (or every) edges of $G$ by an a.g.s. graph, then $K$ has the almost global synchronizing property.

Proposition 4.5: If $G$ is a tree and we build a new graph $K$ replacing some (or every) nodes of $G$ by an a.g.s. graph, then $K$ has the almost global synchronizing property.

These conclusions directly follow from the previous results and are illustrated in figure 7. In [16] it was proved that the complete and the tree graphs are a.g.s., while non a.g.s. graphs, like the cycles with more than 4 nodes, were found. Using this fact, we can prove the following sufficient condition for a.g.s. that partially characterizes the family of all a.g.s. graphs. 


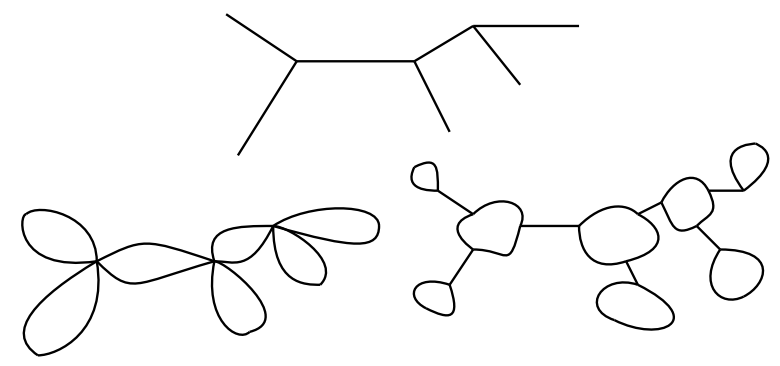

Fig. 7. Situation of Proposition 4.5.

Proposition 4.6: If $G$ is a graph such that all its blocks are complete graphs, then $G$ is a.g.s.

\section{EXAMPLE}

Consider two Kuramoto systems with complete underlying interconnection graphs $G_{1}=K_{3}$ and $G_{2}=K_{5}$ (both a.g.s.). Starting from arbitrary initial conditions, each system quickly reaches a consensus state. At time $T=3$ seconds, we connect the two systems through a bridge between an arbitrary pair of agents. Now, the whole systems reaches a new consensus state. Observe that this convergency is slower than the previous. Figure 8 shows the results obtained from the simulation. They perfectly agree with Proposition 4.2.

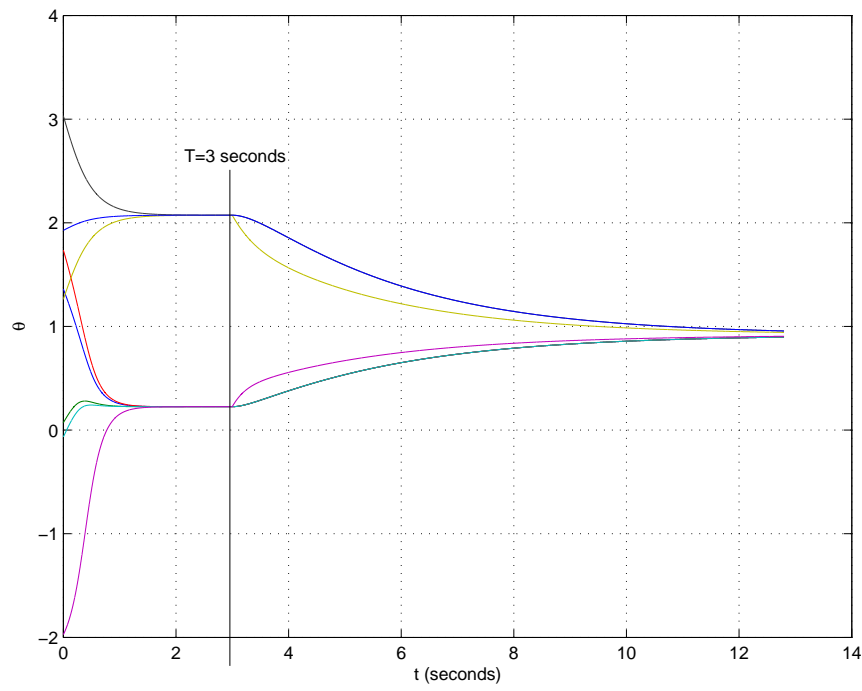

Fig. 8. Two systems connected by a bridge. The connection takes place at time $T=3$ seconds.

\section{CONCLUSIONS}

In this work we have studied how some algebraic properties of the underlying graph describing the interconnection of a symmetric Kuramoto model impose restrictions on the dynamical behavior. In particular, we have tried to advance toward a characterization of the a.g.s. graphs. We focus on the particular case of the existence of a cut-vertex between two subgraphs. We proved that the interconnection by a cutvertex of almost global synchronized systems preserves that property. In particular, we have established that the almost global synchronization analysis of a system with a given interconnection graph $G$ can be reduced to the analysis of the blocks of $G$. In other words, the general a.g.s. problem may be restricted to the analysis of 2-connected graph topologies. This reduction procedure can be also used to glue synchronized systems in order to get a bigger synchronized system. The gluing can be done using cut-vertices or bridges. We have built a family of a.g.s. graphs that includes both the trees and the complete graphs: all whose blocks are complete graphs. We will try to find more a.g.s. classes of graphs and extended the results to Kuramoto models with non sinusoidal interaction functions.

\section{REFERENCES}

[1] Y. Kuramoto, "in international symposium on mathematical problems in theoretical physics," Lecture notes in Physics, vol. 39, p. 420, 1975.

[2] —- "Cooperative dynamics of oscillator community," Progress of Theoretical Physics Suppl, no. 79, pp. 223-240, 1984.

[3] A. Winfree, The geometry of biological time. Springer, 1980.

[4] G. Ermentrout, "The behavior of ring coupled oscillators," Journal of Mathematical Biology, vol. 23, pp. 55-74, 1985.

[5] R. York, "Nonlinear analysis of phase relationships in quasi-optical oscillator arrays," IEEE Trans. on Microwave Theory and Applications, vol. 41, no. 10, pp. 1799-1808, 1993.

[6] S. H. Strogatz, Nonlinear Dynamics and Chaos: with Applications to Physics, Biology, Chemistry and Engineering. Perseus, 1994.

[7] L. Dussopt and J. Laheurte, "Coupled oscillator array generating circular polarization," IEEE Microwave Guided Wave Letters, vol. 9, no. 4, pp. 160-162, April 1999.

[8] S. H. Strogatz, "From Kuramoto to Crawford: exploring the onset of synchronization in populations of coupled nonlinear oscillators," Physica D, no. 143, pp. 1-20, 2000.

[9] A. Jadbabaie, J. Lin and S. Morse, "Coordination of groups of mobile autonomous agents using nearest neighbor rules," IEEE Transactions on Automatic Control, vol. 48, no. 6, pp. 988-1000, 2003.

[10] J.A. Rogge and R.D. Aeyels, "Stability of phase-locking solutions in a ring of unidirectionally coupled oscillators," Journal of Physics A: Mathematical and General, vol. 37, pp. 11 135-11 148, 2004.

[11] J. Marshal, M. Broucke and B. Francis, "Formations of vehicles in cyclic pursuit," IEEE Transactions on Automatic Control, vol. 49, no. 11, pp. 1963-1974, November 2004.

[12] N. Moshtagh, A. Jadbabaie and K. Daniilidis, "Distributed geodesic control laws for flocking of nonholonomic agents," in Proc. of the 44th IEEE CDC and ECC, Sevilla, 2005, pp. 2835-2838.

[13] R. Olfati-Saber, "Swarms on Sphere: A Programmable Swarm with Synchronous Behaviors like Oscillator Networks," in Proc. of the 45th IEEE CDC, San Diego, 2006, pp. 5060-5066.

[14] B.K. Ghosh, A.D. Polpitiya and W. Wang, "Bio-Inspired Networks of Visual Sensors, Neurons,and Oscillators," in Proc. of the IEEE, Vol. 95, No 1 , January, 2007, pp. 188-213.

[15] A. Jadbabaie, M. Barahona and N. Motee, "On the stability of the kuramoto model of coupled nonlinear oscillators," in Proc. of the American Control Conference, 2004.

[16] P. Monzón and F. Paganini, "Global considerations on the kuramoto model of sinusoidally coupled oscillators," in Proc. of the 44th IEEE CDC and ECC, Sevilla, Spain, 2005, pp. 3923-3928.

[17] P. Monzón, "Almost global stabilityt of dynamical systems," Ph.D. dissertation, Udelar, Uruguay, 2006.

[18] P. Monzón and F. Paganini, "Global properties of symmetric couopled oscillators with non complete associated interconnection graph," in Congresso Brasileiro de Automática, Bahia, Brazil, 2006, pp. 470475.

[19] N. Biggs, Algebraic Graph theory. Cambridge University Press, 1993.

[20] D. Cvetkovic, M. Doob and H. Sachs, Spectra of graphs: theory and applications. New York: Academic Press, 1979.

[21] B. van der Pol, "The nonlinear theory of electrical oscillations," Proc. of the Institute of Radio Engineers, vol. 22, no. 9, pp. 1051-1086, 1934.

[22] A. Rantzer, "A dual to Lyapunov's stability theorem," Systems and Control Letters, vol. 42, no. 3, pp. 161-168, March 2001.

[23] H. Khalil, Nonlinear Systems, Prentice-Hall, Ed. Prentice-Hall, 1996. 